\title{
HIGH PRECISION CROWDED FIELD PHOTOMETRY
}

\author{
Peter Linde \\ Lund Observatory \\ Box 43 \\ S-221 00 Lund, Sweden
}

\begin{abstract}
Methods have been developed for high precision photometry in crowded stellar fields. The procedure includes the following steps:

- Determination of a two-dimensional point spread function

- Definition of groups of stars with mutually overlapping images

- Determination of local background for each star group

- Simultaneous fitting of point spread functions, one for each star in the group

The Lund approach emphasizes interactivity. Many fundamental procedures are facilitated by the use of an optimised image display. Extensive modelling has been done to study the influence of various error sources.
\end{abstract}

\section{Derivation of the point spread function}

The Lund method uses a numerical point spread function (PSF), which is derived from the image to be processed. The following steps are taken:

- Selection of stars

- Editing of star images

- Centering of stars

- Normalisation and summation of star images

- PSF quality check

Selection of suitable PSF candidate stars, up to a maximum of twelve, is made by pointing in the stellar field displayed on the screen. The stars are extracted into a set of subimages. In cases where disturbances are seen, single pixel or group pixel editing is performed. Cursors of different geometric shapes are used to identify disturbing pixels. The pixel values are either replaced with a mean of the local neighbourhood or marked for avoidance in following processing steps. The group editing uses a PSF for fitting to neighbouring disturbing stellar images, followed by a subtraction. In this case, the necessary PSF often has to be approximately derived in a previous step.

For star images of typical CCD observations, the sampling is such that subpixel centering is necessary. This step necessitates a resampling of the subimages. The edit and centering 
functions can optionally be done automatically, using an iterative algorithm known as $\kappa-\sigma$ clipping (Newell, 1979). By cross-correlating the selected subinages, pixel values above a preset threshold can be clipped. Recentering and clipping continues until no further changes are introduced.

Remaining pixels in any subimage, differing more than two standard deviations from the mean, are discarded in the summation process. A weight is given to each participating star before summation. Optionally, a filtering algorithm can be applied to the data in order to suppress any remaining noise.

The quality of the resulting PSF is estimated from a careful study of residual images. These are made by subtracting the scaled PSF from the participating individual star subimages. Sometimes, the PSF generation procedure needs to be repeated a few times before the residuals are at a minimum.

\section{Definition of initial star positions}

Although it is attractive to use automatic procedures to locate stars and groups in a crowded field, such detection becomes extremely difficult in very crowded fields. Frequently, faint stars lie in the wings of much brighter stellar images, or conglomerates of faint objects lie near the background noise level. It is clear that undetected group members may seriously affect the measurements of other stars in a group. Therefore, the Lund method emphasizes an interactive approach for very crowded fields. The star position and group definitions are made by pointing in a computer image. The image is displayed on two monitors. One shows a normal grey-scale version and the other shows a pseudo-colour version using a histogram equalised, 16 level, colour sequence. This allows for efficient simultaneous interpretation of both large and small intensity variations in the image.

\section{Determination of the background}

The determination of the background for each measured star is in many cases highly essential for the final precision in the photometry. During the development of the Lund system, several methods have been tried:

- treating the background as a free parameter in the fitting procedure

- interactive determination of local backgrounds for each group

- interactive determination of a background image

- automatic determination from the histogram distribution of the neighbourhood

The first method gave inferior results in very crowded fields due to influence of nongroup members. The second method proved to be too laborious. The last two methods are included in the current algorithms. A background image can be computed using a two-dimensional spline fit to interactively determined background points. The background image is subtracted from the target image. In the final reduction procedure the background then becomes a constant.

Alternatively, an automatic background determination mode is available in the final reduction procedure. Pixel values in a selectable range outside the outer perimeter of each star group are used to compute a background value. Both a clipped mean value and a mode value can be chosen. 
The actual choice of background determination method depends on the degree of image crowding and the background properties in the image. In simple cases, the automatic method works well with either clipped mean or mode values. In more crowded cases, the mode values give better results. In very difficult circumstances, the separately computed background image is the preferred choice.

\section{Fitting the point spread function}

The Lund method uses a multi-fitting technique to calculate position and intensity of each member of a star group. The algorithm creates a model for each group by setting up one copy of the PSF per member star. All PSF:s are then fitted simultaneously, with three free parameters per star. The iteration terminates when residuals between the model and the real group are minimised.

The program runs in two phases. In the first phase, pixels that may disturb the final fitting procedure are identified and excluded by means of dynamic checking of residuals. Such pixels could belong to a wing of some close stellar image, or to a CCD defect. This exclusion procedure is iterated until only relevant pixel data remain.

In the second phase, the final fitting is done using a non-linear least-squares minimising technique. The method is a modified version of the Gauss-Newton algorithm (Gill and Murray, 1978). After each iteration, all variahles are changed simultaneously. The method is unsensitive to initial values of the variables and the convergence is usually fast. The stability is also good, although problems may arise in special circumstances.

\section{Simulations}

In the Lund system, several facilities are available to produce simulated images. With a single command it is possible to produce crowded field test images with arbitrary stellar distributions, PSFs, noise levels, geometries, etc. The stellar magnitude distribution is controlled by astrophysical parameters, such as luminosity functions, colour-magnitude diagrams, etc. Figure 1 shows an example of a simulated $V$ image with realistic noise properties. Figure 2 shows an "ideal" colour-magnitude diagram which would result from a perfect reduction, having photon noise as the only error source in the corresponding $B$ and $V$ images.

The images are subsequently used as input to the crowded field analysis program. A synthetic output image is generated from the results of the program. The difference between the input and the output images, the residual image, is used to examine the quality of the results. An example is seen in Figure 3. Finally, Figure 4 shows an output colour-magnitude diagram, derived using results from both a $\mathrm{V}$ and a $\mathrm{B}$ image reduction. This illustrates the reliability of the reduction procedure in astrophysical terms.

This approach has been used to study different error sources connected to crowded field photometry, including the following:

- different degree of crowding

- image noise

- various PSF sampling levels

- undetected stars

- different PSF shapes 
- different fitting radii

- different background determination methods

Although the various effects are interclependent, efforts were made to separate the effects as much as possible. Thus, optimal conditions for the non-varied parameters were maintained. For example, preknowledge of stellar positions was utilised for the initial coordinates, the PSF used to generate the images was the same used by the analysis program, etc.

\section{Summary of results}

A brief summary of the results will be given here. A full description is available in Linde (1988).

In the first test, analysis was made of noise-free images with increasing degree of crowding. The resulting colour-magnitude diagrams showed almost no increase of spread at the crow ding level illustrated in Figure 1.

Photon noise was then added to the same set of images. Figures 1-4 illustrate this experiment. Some spread, in addition to the expected, is seen in the corresponding colourmagnitude diagram. It should be noted that the data shown in Figure 4 is complete and unedited. From studying residuals in Figure 3, several outliers may be removed. The increase of spread for more crowded fields is larger in the noise case than in the noise-free case. This indicates that coupling exists between increased crowding and increased noise.

Undersampling was tested by using a PSF with a FWHM of 1.8 pixels. Although residuals became slightly more noticeable, the algorithm still worked reliably in the great majority of cases. The main problem for undersampled images is the definition of the PSF.

Undetected stars is the most serious problem in crowded field photometry. A comparison was made between "ideal" coordinate input and the interactive initial coordinate determination method described above. Several stars went unnoticed and showed up in the residual image. The magnitude determinations of neighbouring stars became strongly affected in many cases. However, since their positions were also disturbed, they could often be identified as unreliable in the position matching needed between different exposures. Clearly, many unfavourable cases could be solved by rerunning with improved input data.

A deliberately erroneous PSF was used to test the influence of incorrect PSF shape. The resulting differential image exhibited a considerably increased level of residuals, with a corresponding increase in spread in the colour-magnitude diagram. The main problem revealed by this test was the increased instability of the fitting procedure. In addition, a systematic error in the form of a shift towards brighter magnitudes was noticed.

The test image backgrounds were not complex enough to test the effects of different background estimators. Actually, all three background estimation methods, discussed in section 3 , worked equally well.

\section{Speed and positional accuracy}

The computer used was a Hewlett-Packard $9000 / 530$ with floating point accelerator. For groups with less than five members, the computing time was about 0.5 minutes per star. For a group with fifteen stars, the time rises to four minutes per star. Although the program allows a large number of stars per group, it is currently not practical to have more than 
twenty at a time. As a byproduct of the reductions, precise star positions are also determined. For images with typical noise, about $70 \%$ of correlated position determinations lie within 0.05 pixels.

\section{Comparison with other methods}

Several algorithms for crowded field photometry have been described in the literature. These include the ROMAPHOT package (Buonanno et al., 1983), algorithms by Penny (1976) and Irwin (1986), which all utilise analytically defined PSFs. The DAOPHOT package (Stetson, 1987 ) uses an approach similar to the Lund method regarding the derivation and usage of numerical PSFs. However, in very dense fields, automatic algorithms tend to give misleading results. This is where interactive elements are of greatest importance. The Lund algorithms provide interactivity in the most critical phases of crowded field photometry. They include careful preparation of the input data as well as qualitative (visual) and quantitative quality checks of the output data.

\section{References}

Buonanno, R., Buscema, G., Corsi, C.E., Ferraro, I. and Iannicola, G., Astron. Astrophys, 126, p. 278, 1983.

Gill, P.E., and Murray, W. SIAM Journal on Numerical Analysis, 15, p. 97̄, 1978.

Irwin, M.J., In Data Analysis in Astronomy, Eds. di Gesù, V., Scarsi, L., Crane, P., Friedman, J.H., Levialdi, S., Plenum Press, New York-London, p. 439, 1985.

Linde, P., Image analysis in Astronomy, thesis, Lund Observatory 1988.

Newell, B., In Image Processing in Astronomy, Eds. G. Sedmak, M. Cappaccioli, R.J. Allen, Osservatorio Astronomico di Trieste, p. 100, 1979.

Penny, A.J., In IAU Colloquium 40, Astronomical Applications of Image Detectors with Linear Response, eds. M. Duchesne and G. Lelievre (Paris: Paris-Meudon Observatory), p. $49-1,1976$.

Stetson, P.B., Publ. Astron. Soc. Pacific, 99, p. 191, 1987. 


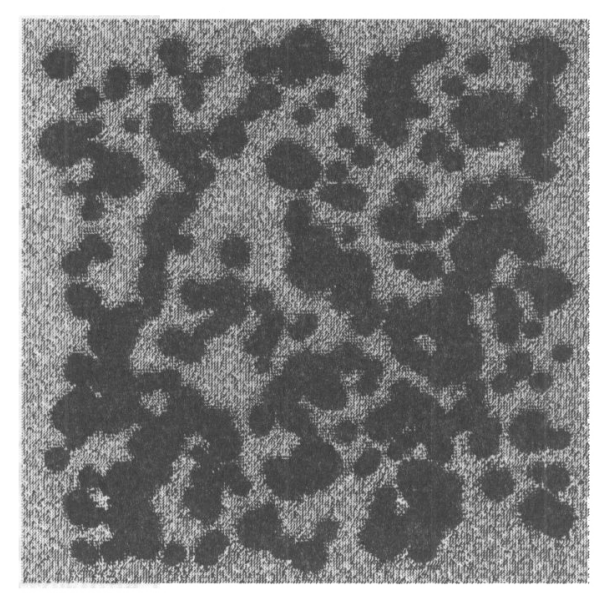

Figure 1. An artificial V field with 337 stars. Image size is $200 * 200$ pixels. Noise according to Poisson statistics has been added. The FWHM size of the point spread function used for generating the stars is 3.6 pixels.

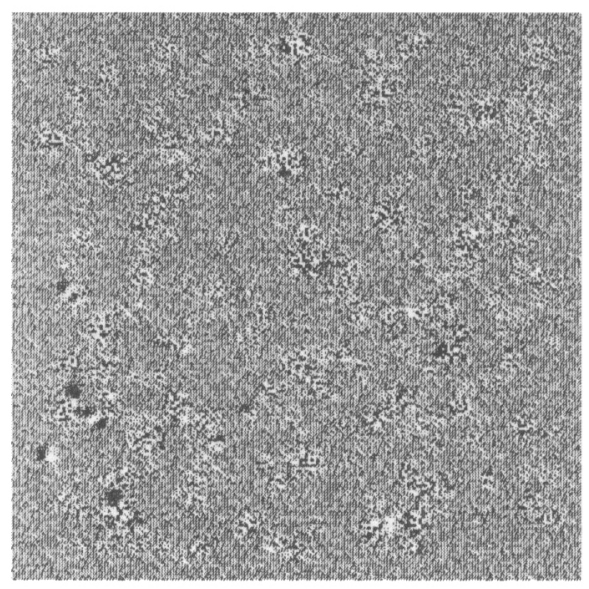

Figure 3. A differential image showing residuals between the field shown in Figure 1 and the measured data.

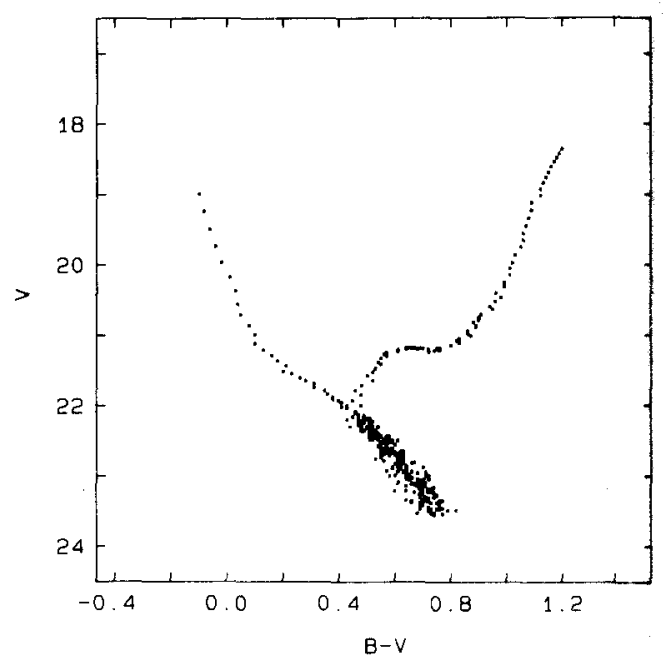

Figure 2. A colour-magnitude diagram as it would appear if the image shown in Figure 1 (and its B image counterpart) had been re. duced with no error source except the photon noise.

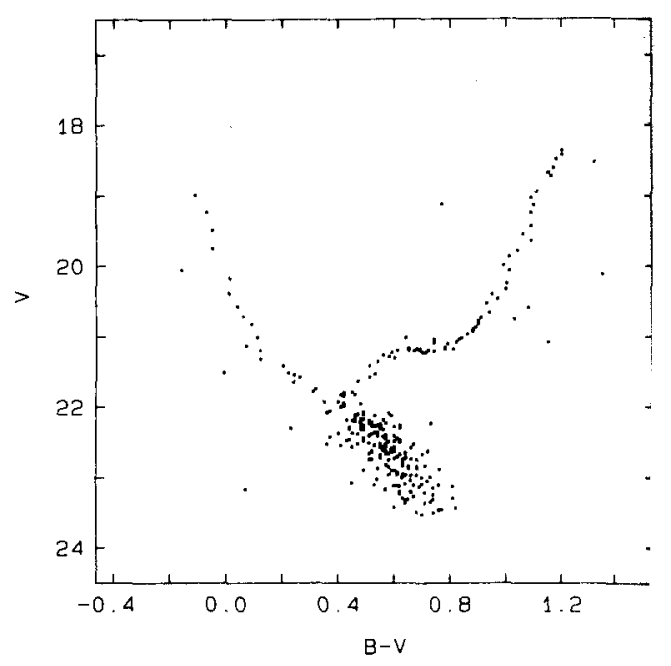

Figure 4. The resulting (raw) colour-magnitude diagram from analysis of the image shown in Figure 1 (and its B counterpart). 